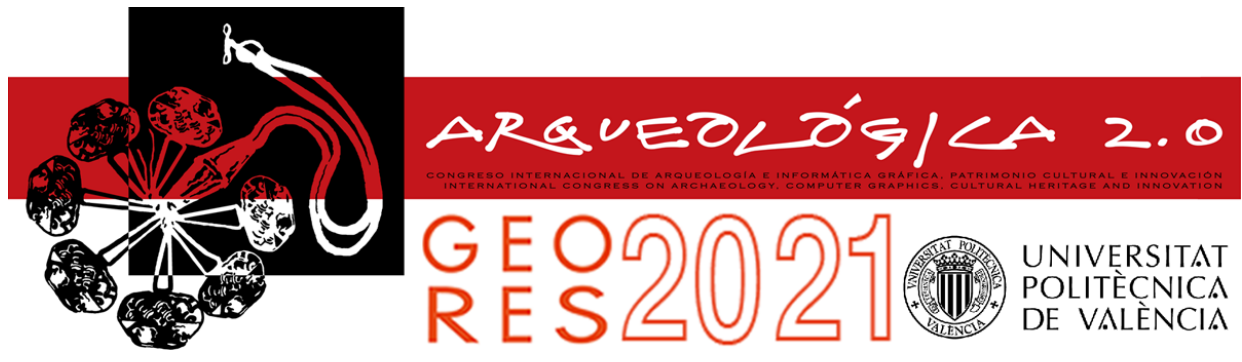

Proceedings of the joint international event $9^{\text {th }}$ ARQUEOLÓGICA

$2.0 \& 3^{\text {rd }}$ GEORES

Valencia (Spain).

26-28 April 2021

\title{
TOWARDS AN INTEGRATED DESIGN METHODOLOGY FOR H-BIM
}

\author{
Eugenio Pellis a, ${ }^{a}$, Andrea Masiero ${ }^{a}$, Grazia Tuccia ${ }^{a}$, Michele Bettia, Pierre Grussenmeyer ${ }^{b}$ \\ a Department of Civil and Environmental Engineering, University of Florence, Via di Santa Marta 3, 50139 Florence, Italy. \\ eugenio.pellis@unifi.it; andrea.masiero@unifi.it; grazia.tucci@unifi.it; michele.betti@unifi.it \\ ${ }^{b}$ Photogrammetry and Geomatics Group, ICube Laboratory UMR 7357, INSA Strasbourg, University of Strasbourg, France. \\ pierre.grussenmeyer@insa-strasbourg.fr
}

\begin{abstract}
:
In recent years, the numerous advantages introduced by Building Information Modelling (BIM) have led to its application on the heritage environment and giving birth to the concept of H-BIM (Heritage BIM). The resulting demand in heritage survey data processing has focused this research on the development of strategies and methods to improve the construction of three-dimensional and informative models starting from 3D point clouds. The implementation of an automated procedure is fundamental for easing and speeding up the survey data processing and one of the most challenging tasks to achieve this purpose is the problem of semantic segmentation. The research presented in this paper aims at testing already existing methods and exploring new strategies for 3D point cloud semantic segmentation on heritage scenarios focusing on deep learning and neural network techniques.
\end{abstract}

Keywords: H-BIM, cultural heritage, 3D point clouds, semantic segmentation, deep learning

\section{Introduction}

Over the last few decades three-dimensional (3D) modelling, in particular, the Building Information Modelling (BIM), has become a standard design approach in the Architecture, Engineering and Construction (AEC) sector (Azhar, 2011; Azhar, Khalfan \& Maqsood, 2012). While for new buildings the design methodologies are quite well defined, the BIM platforms still have serious issues to represent historical buildings and cultural heritage. From a geometrical point of view, heritage elements, due to their uniqueness, irregularity, and shape complexity, require careful modelling. From an informative aspect, a proper comprehension and description of heritage buildings requires heterogeneous information like images, drawings, report, information from previous surveys, etc. As a matter of facts, given such a complex set of information to be properly integrated, regular software and databases are not suitable for dealing with this kind of application (Oreni, 2013; Khalil \& Stravoravdis, 2019; Dore \& Murphy, 2017).

These challenges lead to the need for the development of an ad hoc approach for historical buildings and to the concept of Heritage Building Information Modelling $(\mathrm{H}$ BIM) (Murphy, McGovern \& Pavia, 2009). Over the last few years, the growing interest for H-BIM turns out from the numerous advantages of this type of modelling, and it goes along with the growing necessity of protection, conservation, restoration, and dissemination of cultural heritage (López et at., 2018; Volk, Stengel \& Schultmann,
2014). A well-established approach for the construction of an as-built model starts from data acquisition, the processing of point clouds, up to the construction of a three-dimensional (3D) and informative model (Fig. 1). This workflow is commonly called Scan-To-BIM (Rocha, Mateus, Fernández, \& Ferreira, 2020; Laing, Leon, Isaacs, \& Georgiev, 2015). Nowadays, the high levels of detail and automation achieved by 3D laser scanning and photogrammetry technologies allow to collect of a large amount of data in a short time (Grussenmeyer, Landes, Voegtle, \& Ringle, 2008), but properly processing such data is still a quite long procedure, requiring a time consuming manual intervention by a specialized operator, aiming at the definition of a 3D parametric model of the building of interest suitable for standard BIM software. (Rodriguez-Moreno et al., 2016; Tang, Huber, Akinci, Lipman, \& Lytle, 2010).

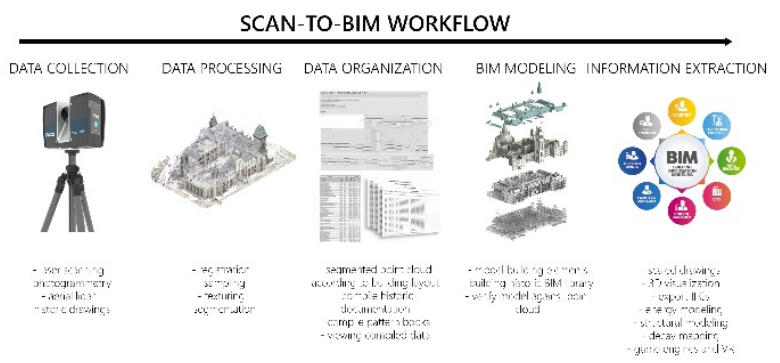

Figure 1: Scan-To-BIM workflow. 
One of the key points to enable automatic data processing in the above-mentioned procedure, is the development of a proper automatic semantic segmentation procedure (Macher, Landes \& Grussenmeyer, 2015). Semantic segmentation is the process of classifying each pixel in an image or each point in a 3D point could into classes or categories that share similar feature characteristics (Fig. 2). In practice, it can be considered as a fundamental step in the machine automatic comprehension of the considered data, and hence it plays a central role in a wide range of applications such as scene understanding, robotic perception, autonomous driving, augmented reality, and many others. The continuously increasing number of applications employing semantic segmentation makes it a very active research field, with several segmentation approaches already proposed in the literature (Yu et al., 2018; Nguyen \& Le, 2013).

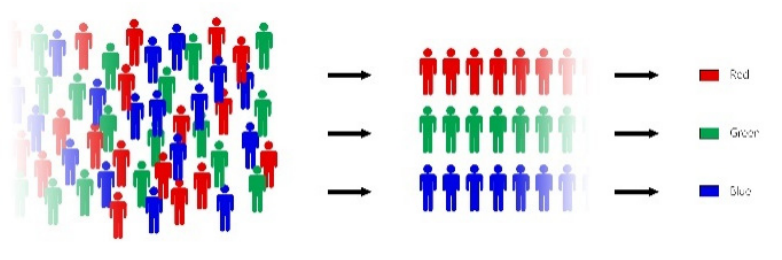

Figure 2: The concept of semantic segmentation.

Given the demand for automatic data analysis and modelling that emerged during the last decade also in the Architecture, Engineering and Construction (AEC) sector, many studies have explored strategies to apply semantic segmentation to the AEC problems as well. However, in particular for historical buildings, there is still a lack of standard procedures and methodologies to manage such complex data, and currently, the results are still not sufficiently accurate to fully understand heritage scenes and automatize the creation of detailed three-dimensional models (Grilli \& Remondino, 2019; Grilli, Menna \& Remondino, 2017).

The research presented in this article aims at partially reducing the above-mentioned lack, testing already existing methods, and exploring new strategies and methods for point cloud semantic segmentation in heritage and historical scenarios. Furthermore, this research also contributes to the generation of a database to be used as a benchmark for comparing different heritage building segmentation approaches: such database can be considered both as integration of certain already existing ones, and as a new one, providing the possibility of investigating point cloud segmentation methods and multi-view based ones as well.

\section{Semantic segmentation review}

3D point cloud semantic segmentation (PCSS) is attracting an increasing interest due to its applicability in a wide range of different applications (Xie, Tian \& Zha, 2020). Despite the term semantic segmentation is widely used in computer vision, in photogrammetry and remote sensing applications, the following nomenclature is also often used for similar purposes: "point cloud classification" or "point labelling" (Boulch, Le Saux \& Audebert, 2017).

Given a point cloud, the goal of semantic segmentation is to separate it into several subsets according to the semantic meaning of the points. Artificial Intelligence (Al), in particular the branch of machine learning, has become the basic building block for these tasks and nowadays PCSS is usually realized by supervised learning methods, including "regular" supervised machine learning and deep learning (Guo et al., 2020).

\subsection{Regular Supervised Machine Learning}

Regular supervised machine learning methods for semantic segmentation of point clouds can be divided into two main groups (Weinmann et at., 2015):

1) Individual PCSS methods, which classify each point based only on its individual features. Four stages can usually be identified in these methods: neighbouring selection, feature extraction, feature selection, and semantic segmentation. These methods are usually computationally efficient, but their results are often affected by a significant level of noise. The most important methods in this category are Random Forest, AdaBoost, Support Vector Machine, Maximum Likelihood.

2) Methods based on statistical contextual models, which focus on point cloud statistic and relational information over different scales. Differently from individual PCSS they take into account contextual features. The most widely used model in this category is Conditional Random Fields (CRF) (Weinmann, Jutzi, Hinz, \& Mallet, 2015; Vosselman, Coenen \& Rottensteiner, 2017).

\subsection{Deep learning}

Deep learning has been recently successfully used on several 2D vision problems, becoming more and more popular during the last five years, in particular after the introduction of Convolutional Neural Networks (CNN) (He, Zhang, Ren, \& Sun, 2016), and nowadays it can be considered as a predominating technique in Al.

Given the typical high performance of deep learningbased solutions, there is an increasing interest of the civil engineering sector in the extension of the use of such techniques also to data related to construction and building models. For instance, they may be used to extract information from 3D point clouds, for 3D Shape classification, 3D Object Detection, 3D Object Tracking, and 3D Point Cloud Segmentation (Zhu et al., 2017; Long, Shelhamer \& Darrell, 2015).

Three-dimensional data provide richer spatial and geometrical information compared to two-dimensional data and could better characterize complex scenes. However, the use of deep learning methods on point clouds still faces several significant challenges, due for instance to:

- The large data size, which implies long computing time,

- The unstructured nature of 3D point clouds, which complicates the use of network architectures commonly used for 2D data,

- The unavailability of large shared datasets, which makes the results of the training process hard to be exportable to scenarios different from the one that motivated the network realization. 
According to the literature, semantic segmentation methods for $3 \mathrm{D}$ point cloud can be divided into two groups: a) projection-based methods and b) point-based methods (Zhang, Zhao, Chen, \& Lu, 2019), which are going to be described in the following.

\subsubsection{Projection-based methods}

The main issues to be solved for using standard neural networks, such as Convolutional Neural Networks (CNNs) or Fully Connected Layers (FCs), are the unstructured nature of point clouds and the presence of orderless data. To this aim, projection-based methods first apply a transformation to convert 3D point clouds on a data with a regular structure, then they perform the semantic segmentation task by applying standard approaches, and finally, they re-project the extracted features on the starting shape or point cloud (Lawin at al., 2017). The advantage of projection-based methods is that they leverage well-established networks. However, any kind of transformation and intermediate representation involves inevitably a loss of information, in particular geometrical and spatial. Depending on the type of used representation, it is possible to distinguish four categories among these methods: multi-view, volumetric, spherical, and lattice.

\subsubsection{Multi-view representation}

These methods first project the 3D shape into multiple views, then apply $2 \mathrm{D}$ image segmentation methods to extract information from each image. The results obtained on such images are compared and analysed, and eventually re-projected on the original scene to obtain a semantically segmented point cloud. How to aggregate the multiple views in a global representation is still a key challenge for this method.

MVCNN (Su et al., 2015) is a pioneering work, which proposed the use of Convolutional Neural Networks (CNN) with multiple perspectives of the $3 \mathrm{D}$ object. It is suitable for an individual object rather than complex scenes because it ignores the spatial relationship between objects. Another important work is SnapNet (Boulch, Guerry, Le Saux, \& Audebert, 2018), that, in order to address the problem of information losing, it selects some snapshots of the point clouds to generate RGB and depth images, and then it uses the marked points to project the segmentation on the $3 \mathrm{D}$ cloud. The more recent SnapNet-R improves the process of image generation and the overall accuracy. These networks ensure excellent image segmentation results, but the transposition of such results on the 3D cloud entails a large loss of spatial and geometrical information.

\subsubsection{Volumetric representation}

Volumetric representation or voxelization of point clouds consists in the transformation of the unstructured 3D cloud into a regular spatial grid, and then the information distributed on such a regular grid is exploited to train a quite standard neural network to properly perform the segmentation task. VoxNet (Maturana \& Scherer, 2015) converts the $3 \mathrm{D}$ clouds into a grid in which CNN operations can be applied and use CNN to predict the classes directly on the order grid. PointGrid uses the same transformation of VoxNet, but it addresses the problem of information loss and change of scale, and it has less memory requirements. SEGCloud (Tchapmi, Choy, Armeni, Gwak, \& Savarese, 2017), to reduce the computational cost has introduced the methods of spatial partition such as K-d tree or Octree. In conclusion, the mentioned methods and others like OctNet (Riegler, Ulusoy, \& Geiger, 2017), VV-Net (Meng, Gao, Lai, \& Manocha, 2018), ScanComplete (Dai et al., 2017) ensure the achievement of a reasonable segmentation of nonstructured relatively small point clouds. Unfortunately, they are still unsuitable for the semantic segmentation of complex scenarios.

\subsubsection{Spherical representation}

These types of representation, compared with the multiview representation, retain more geometrical and spatial information. However, they have some issues such as discretization errors and occlusion. The most important works are SqueezeNet (landola et al., 2017) and RangeNet++ (Milioto, Vizzo, Behley, \& Stachniss, 2019) for real-time LiDAR data semantic segmentation.

\subsubsection{Lattice representation}

Volumetric representation is naturally sparse, and it is inefficient to apply dense convolutional neural networks (DCNN) on spatially sparse data. Lattice representation converts a point cloud into discrete representation such as sparse permutohedral lattice. This method can control the sparsity of the extracted features and reduces memory requirements and computational costs reducing the convolution output. One of the main works is SPLATNet (Su et al., 2018). It interpolates a raw point cloud to a sparse lattice and then a Bilateral Convolutional Layers (BCL) is applied to convolve on occupied parts of lattice. Other works are LatticeNet (Rosu, Schutt, Quenzel, \& Behnke, 2017), which achieves efficient processing of large point clouds, and MinkowskiNet (Choy, Gwak \& Savarese, 2018), a 4D spatio-temporal convolutional neural network for $3 \mathrm{D}$ video perception.

\subsubsection{Point-based methods}

Point-based methods, or direct methods, work directly with point clouds and they do not introduce explicit information loss with intermediate representations. This direct approach leverage on the full use of the characteristic of the raw point cloud data and consider all the geometrical and spatial information. Despite pointbased methods are still in development, they seem the most promising in the future and a series of networks have been proposed recently. Overall, these methods can be divided into four categories: pointwise MLP methods, convolution methods, RNN-based methods and graphbased methods.

\subsubsection{Pointwise methods}

These methods usually use shared Multi-Layer Perceptron (MLP) as the basic unit in their network. The pioneering work for this method is PointNet (Qi, Su, Mo, \& Guibas, 2017), it learns per-point features using shared MLPs and global features using symmetrical polling functions. However, MLP cannot capture local geometry in the mutual interaction between points. In order to capture wider context and learn more local structures, a lot of network-based on PointNet have been developed recently. These methods are based on neighbouring 
feature pooling such as PointNet++ (Qi, Yi, Su, \& Guibas, 2017), PointSIFT, PointWeb, RandLA-Neton attentionbased aggregation such as Gumbel Subset Sampling (GSS) or Local Spatial Aware (LSA), and on local-global concatenation such as EdgeConv and NetVLAD.

\subsubsection{Convolution methods}

These methods tend to propose effective convolution operators for point clouds (Hua, Tran \& Yeung, 2018). PointCNN (Wang et al., 2018) is a network-based on parametric continuous convolution layers and the kernel function of this layer is parametrized by MLPs. KP-FCNN is based on Kernel Point Convolution (KPConv), and the convolution weights are determined by the Euclidean distances to kernel points, and the number of kernel point is not fixed. ConvPoint proposed a point-wise convolution operator, where the neighbouring points are binned into kernel cells and then convolved with kernel weights.

\subsubsection{RNN-based methods}

Recently Recurrent Neural Network (RNN) have been used for semantic segmentation, in particular, to capture inherent context features from point clouds. $\mathrm{G}+\mathrm{RCU}$ first transformed a block of points into multi-scale blocks and grid blocks to obtain input-level context. Then, the blockwise features extracted by PointNet are sequentially fed into Consolidation Units (CU) or Recurrent Consolidation Units (RCU) to obtain output-level context. 3DCNN-RNN (Liu et al., 2017) first learns spatial distribution and colour features using a 3D CNN, and then the final concatenated feature vector is fed into a residual RNN to obtain the final segmentation. However, these methods lose geometric features and density distribution from point clouds when aggregating the local neighbouring features with global structures.

\subsubsection{Graph-based methods}

To improve the results and capture richer geometrical structures several methods leverage graph networks. Graph Neural Network (GNN) is a type of Neural Network which directly operates on the Graph structure. The most important works are DGCNN (Wang et al., 2018), PyramNet based on Graph Embedding Module (GEM), and GACNet.

Finally, a summary of the main network architectures with their typology, year, and accuracy (mloU) on the ModelNet40 dataset (Fig. 3).

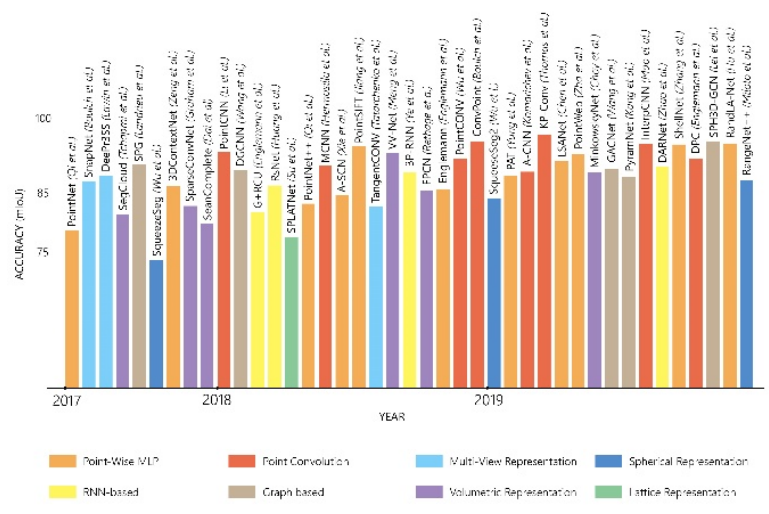

Figure 3: Summary of the main networks for 3D point cloud semantic segmentation.

\section{Research goals}

According to many studies, the problem of managing and processing $3 \mathrm{D}$ point cloud data is still a challenging task, in particular 3D complex data such as the historic and heritage ones. The main aim of this research is to develop strategies and methods to improve the automatization of 3D point cloud data processing and further the construction of three-dimensional models. As mentioned above, one of the key points for these purposes is $3 D$ point cloud semantic segmentation and this research has the purpose to investigate methods and techniques to improve segmentation on heritage 3D point clouds. Among all the methodologies describe previously, the research is focused on Deep Learning and Neural Networks techniques.

The choice of these typologies is motivated by their growing use in various applications, the great number of different algorithms and the continuous spread of new techniques, that could be adapted and tested on heritage 3D data. Despite the algorithm, results are improved every year, and new methods are proposed to improve their accuracy and reliability, there are few works that are focused on the use of Deep Learning on heritage data (Llamas, M. Lerones, Medina, Zalama, \& Gómez-GarcíaBermejo, 2017; Matrone et al., 2020a; Heipke \& Rotternsteiner, 2020).

This work aims at investigating two different approaches:

- Multi-view approach, based on image semantic segmentation and then on the re-projection of the extracted features on the point cloud,

- Point-based approach, that works directly with the raw point cloud.

The multi-view approach is being investigated first, mostly motivated by the greater simplicity and clarity of image-based algorithms and by the strong and solid results obtained recently on image segmentation (Minaee et al., 2020; Ulku \& Akagunduz, 2020). In addition, at this time, a multi-view approach for semantic segmentation on heritage data has never been tested and it is reasonable to also explore this direction. However, the results with point-based methods are improving rapidly and, in our future research work, this approach will be tested as well. The construction and the development of a specific network for heritage segmentation needs necessarily of a dedicated dataset for training and validation step and currently there are not available datasets for this purpose. The creation of a good dataset is a long and timeconsuming procedure, but this effort is motivated by the possibility of using it for comparing a) various network architectures and b) point-based and multi-view approaches, c) for possible integration with point-based methods, and hence for the development of a hybrid image-point based method that may improve performance, accuracy and reliability of the existing methods.

To recap, the main goals of the first phase of this research are two: a) the construction of a dedicated dataset for semantic segmentation of heritage buildings and $b$ ) the testing and tuning of image segmentation algorithms on this specific dataset. 


\section{Dataset creation}

Datasets play an indispensable role during the model training, and a large number of datasets have been created to evaluate the performance of deep learning algorithms for different applications.

In the context of 3D point cloud semantic segmentation, the most used and complete datasets are Paris-Lille-3D, ScanNet, Semantic3D (Hackel et al., 2017), SemanticKITTI, DALES (Varney, Asari, \& Graehling, 2020). In the specific context of heritage and historical environment, only two datasets are available. The first is Architectural Heritage Elements Dataset (AHE_dataset), a collection of 10,000 images classified in 10 types of architectural elements for the task of images classification. The second is ARCH_dataset (Matrone et al., 2020b), a benchmark for large-scale heritage point cloud semantic segmentation, composed of 17 annotated scenes in 10 categories.

The new dataset will be structured following the categories and the guidelines of $\mathrm{ARCH}$ dataset. The images will be a label with 10 classes including wall, floor, roof, column, moulding, vault, arch, stair, window/door and others, corresponding with the BIM standard constructive elements. An example of a segmented image according to ARCHdataset is shown below (Fig. 4). This choice, in addition to the possibility to compare the progress and the obtained results, allows a possible collaboration with the $\mathrm{ARCH}$ dataset. On one hand, introducing different scenes and increasing the number and the typologies of the labelled buildings. On the other hand, introducing the possibility of integration with photogrammetry images that are often paired with LiDAR data. This would entail the creation of a richer and more complete dataset, improving the generalization and the learning capability and the possibility to develop new algorithms and improve the existing ones.

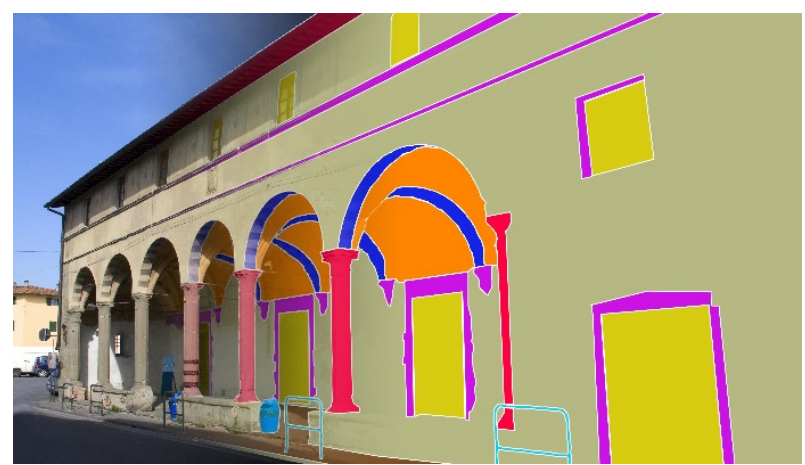

Figure 4: Example of a segmented image according to ARCHdataset.

Initially, the dataset will be composed of 9 partial scenes of heritage buildings from different historical period and architectural styles. The survey data were collected in research and didactical context by GECO Laboratory. GECO, affiliated with the Department of Civil and Environmental Engineering of Florence (DICEA), is a Geomatics and Conservation laboratory, headed by prof. Grazia Tucci. Its activities are mainly focused on documentation, protection, and enhancement of Cultural Heritage with innovative technologies. For all these buildings the laboratory has already available the LiDAR data that will be integrated, for the creation of the dataset, with terrestrial photogrammetry images. Due to the limitations of the latter, the scenes will be probably incomplete: they will mostly include façades and loggia.

The presence of the two data types allows to exchange of features and information between photogrammetry and LiDAR clouds, facilitate and semi-automatize the segmentation operation to obtain the ground truth and speed up the creation of the dataset. Further, both point clouds could be used with a point-based network.

A list and the images (Fig. 5) of the nine buildings that will be used for the creation of the dataset is reported below.

1) Spedale di Sant' Antonio, Lastra a Signa

2) Ospedale del Ceppo, Pistoia

3) Palazzo Pitti, Firenze

4) Basilica della Santissima Annunziata, Firenze

5) Ca' Granda, Milano

6) Galleria dell' Accademia, Firenze

7) Certosa del Galluzzo, Firenze

8) Cappella Buontalenti, Firenze

9) Palazzo Vecchio, Firenze
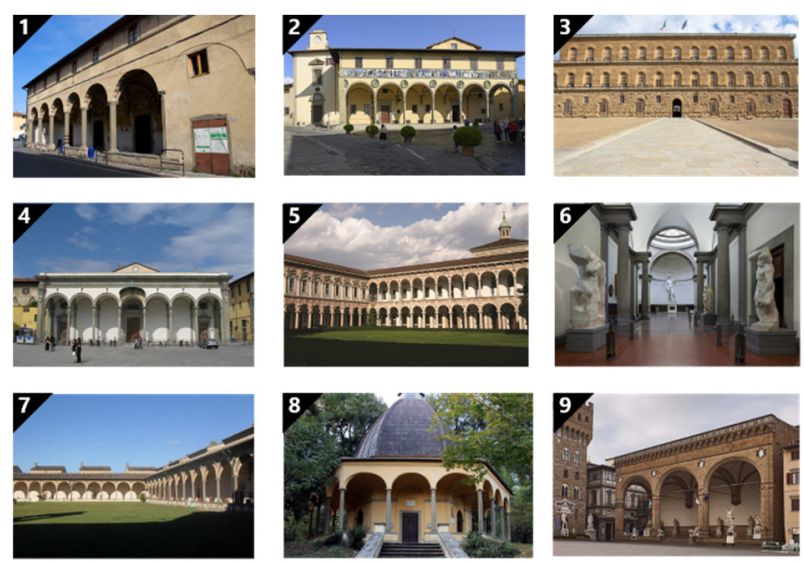

Figure 5: The nine buildings of the dataset.

A good and functional image-based dataset should be composed of thousands of images and a semiautomatized procedure is crucial to obtain results in a reasonable time. As shown below a possible solution tested with the façade of SS Annunziata case study is reported. Starting from survey images (1), with the standard software tools a dense point cloud was built (2). After pre-processing operations such as cleaning, sampling and alignment with the LiDAR cloud, the point cloud was manually segmented (3). Finally, with the last step, the features were reprojected on the initial images obtaining all the images segmented (4) (Fig. 6).

The longest and most challenging step is the third, but a semi-automatize process will be developed and tested. However, the effort could be justified by three reasons: (i) the obtained clouds could be used to quickly project the features also on LiDAR clouds, (ii) through only one cloud it is possible to project the segmentation to hundredsthousands of images and (iii) both could be used also as inputs of point-based networks.

Once the procedure will be performed on all the proposed buildings, the initial number of the labelled images in the dataset will be around 5000-7000 images. Although the many images are just enough for the initial tuning of the network and the size of the dataset will be probably not 
enough to train from scratch a network for a complex task such as semantic segmentation. In addition, to increase the number of the study case, two methods can be used to face this problem: (i) data augmentation, that allows increasing the number of the image just mirroring, cutting and filtering the images, and (ii) synthetic data generation, that allows to automatically create new data starting from a data source.
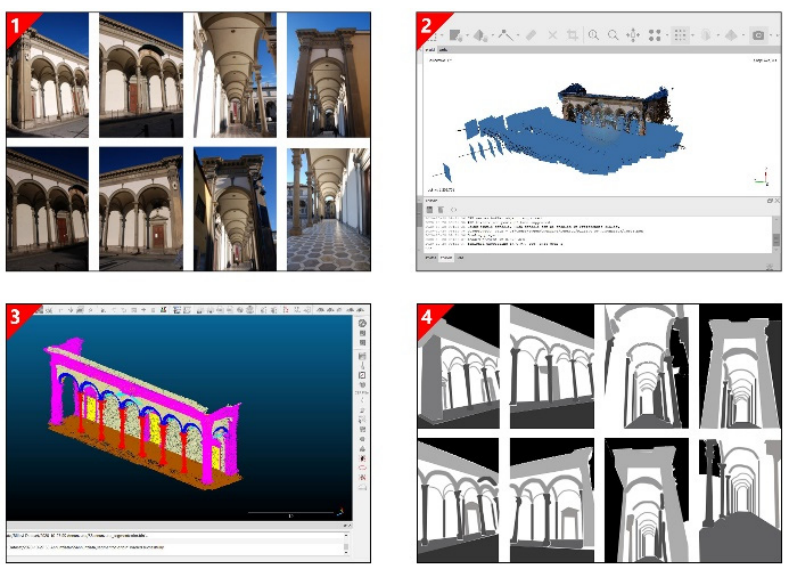

Figure 6: Four steps for dataset creation.

\section{Semantic segmentation methods}

Once the dataset will be created, the research will focus on the implementation and testing of various existing neural network architectures for image semantic segmentation on the new data. Numerous algorithms have been developed in the literature for this task, like region-growing (Nock \& Nielsen, 2004), K-means clustering (Dhanachandra, Manglem, \& Chanu, 2015), sparsity-based methods (Minaee \& Wang, 2019) and others. Over the last past few years deep learning networks, usually Convolutional Neural Networks (CNNs), have reached remarkable performance, surpassing other approaches by a large margin in terms of accuracy and efficiency (Garcia-Garcia et al., 2018).

The task of semantic segmentation is the natural extension of simpler problems: at first classification, which consists of making a prediction of a whole input, and then localization or detection, which regard the spatial location of the objects. The next step of semantic segmentation is to make a dense prediction inferring labels for every pixel. For this reason, common networks approach for classification are often used as building blocks for many semantic segmentation architectures, like AlexNet (Krizhevsky, Sutskever \& Hinton, 2012), VGG (Simonyan \& Zisserman, 2014), GoogleNet (Szegedy et al., 2015), ResNet (He, Zhang, Ren, \& Sun, 2016).

Currently, the most successful state-of-art deep learning techniques stem from a common forerunner: the Fully Convolution Network (FCN) (Long, Shelhamer \& Darrell, 2015). They transform the existing and well-known classification models into FCN replacing the fully connected layers with convolutional ones to output spatial maps with per-pixel labelling instead of classification scores. Despite its popularity and flexibility, FCN has some limitations. It is not fast enough, it does not take into account useful global context information and it is not well suited for unstructured data. To address these problems other methods and architecture have been proposed.
Among Fully Convolution-based methods, ParseNet (Liu, Rabinovich, \& Berg, 2015) adds global context by using the average feature for a layer to augment the feature at each location. Some popular deep models are based on encoder-decoder architecture. Two popular works are SegNet (Badrinarayanan, Kendall, \& Cipolla, 2015) and U-Net (Ronnenberg, Fisher \& Brox, 2015). SegNet is composed by a set of upsampling and convolutional layers which are at last followed by a softmax classifier to predict pixel-wise labels. U-Net was initially proposed for biomedical image and then used also for aerial images. It consists of a contracting path (downsampling) that captures context and a symmetrical expanding path (upsampling) that enables precise localization. Dilated or "atrous" convolution-based methods allow efficient dense feature extraction on any resolution. The most important works include DeepLab family (Chen, Papandreou, Kokkinos, Murphy, \& Yuille, 2017), Multi-scale Context Aggregation (Yu \& Koltun, 2015) and ENet (Paszke, Chaurasia, Kim, \& Culurciello, 2015). Recently also RNNs based models were proposed. Using RNNs, pixels may be linked together and processed sequentially to model global contexts and improve semantic segmentation. Two important works that leverage this architecture are ReSeg (Visin et al., 2016), based on the classification network ResNet, and LSTM-CF (Li, et al., 2016), which use two different data sources, RGB and depth.

Between the most popular state-of-the-art techniques the research will be focus on the implementations of two networks: (i) U-Net and (ii) DeepLabv3+. The choice of these architectures is motivated by their remarkable results on different data sources and by their popularity, which makes documentations and shared implementations easily available. In a first step, the dataset will be split in a training and a validation set. Secondly, according with the training and validation results, the network will be improved tuning the hyperparameters, trying to prevent overfitting or underfitting, and possibly changing some building blocks of the network to make it fit with the new data. Depending on the obtained results, other networks may be tested.

Training a deep neural network from scratch is often unfeasible for various reasons: a dataset of sufficient size is required and reaching convergence can take too long for the initial tuning of a network. For these reasons two techniques will be used to test the performance on the new data: (i) transfer learning and (ii) data augmentation.

Transfer learning is a technique that allows to grab a model trained on a certain task and dataset, and employ it on a different task. As a consequence of such re-use of the original network, the learning procedure for the new one starts with pre-trained weights instead of randomly initialized ones. Fine-tuning the weights of a pre-trained network by continuing with the training process is one of the major transfer learning scenarios. In the context of semantic segmentation, the difficult of gathering and creating per-pixel labelled datasets that make their scale not large and the learning procedure from scratch difficult, enhances transfer learning a standard procedure. The most popular and largest dataset for semantic segmentation is ImageNet, and due to its size and completeness it is often use to pre-train new networks.

Data augmentation is a common technique used for training machine learning models and consists of applying a set of transformations in the training data such as 
translation, rotation, warping, mirroring, scaling, crops, colour shifts etc. The aim of these transformations is to increase the number of the images and to create a larger dataset. Thanks to augmentation, which is especially helpful when the available dataset is small, it is possible to partially prevent overfitting, increase generalization capabilities, regularize the model, balance the classes, and speed up the convergence. For instance, augmenting a dataset of 1000 images with four rotations, four new scales, and four gamma variations, it is possible to generate a new dataset of 13000 training images.

\section{Conclusions}

This paper has shown the motivations and purposes of my $\mathrm{PhD}$ research, focused on the problem of 3D semantic segmentation of heritage scenarios. Starting from a multi- view approach up to point-based methods, the aim of this research is to test existing deep learning algorithms on heritage survey data. Actually, the main goals are two. First, the creation of a dedicated dataset for image semantic segmentation of heritage buildings with a semiautomatic approach. Second, the implementation of existing network architecture and the testing on the new data source. Currently a semi-automatic procedure for the creation of the dataset was created and based on next test, it will be improved. The future goals of the research are to integrate the new dataset with the existing ones, creating a benchmark with more generalization capabilities based both on images and points, and the exploration and development of point-image based networks that could increase the overall performance on heritage scenarios.

\section{References}

Azhar, S. (2011). Building Information Modeling (BIM): Trends, Benefits, Risks, and Challenges for the AEC Industry. Leadership and Management. Engineering, 11(3), 241-252. https://doi.org/10.1061/(ASCE)LM.1943-5630.0000127.

Azhar, S., Khalfan, M., \& Maqsood, T. (2012). Building information modelling (BIM): now and beyond. Australasian Journal of Construction Economics and Building, 12(4), 15-28. https://doi.org/10.5130/AJCEB.v12i4.3032.

Badrinarayanan,V., Kendall, A., \& Cipolla, R. (2015). Segnet: a deep convolutionalencoder-decoder architecture for scene segmentation. IEEE Trans. PatternAnal. Mach. Intell., $39 \quad$ (12), 2481-2495. https://doi.org/10.1109/TPAMI.2016.2644615

Boulch, A., Le Saux, B., \& Audebert, N. (2017). Unstructured point cloud semantic labeling using deep segmentation networks. 3DOR. http://dx.doi.org/10.2312/3dor.20171047

Boulch, A., Guerry, J., Le Saux, B., \& Audebert, N. (2018). Snapnet: 3d point cloud semantic labeling with 2d deep segmentation networks. Computers \& Graphics, 71, 189-198. https://doi.org/10.1016/j.cag.2017.11.010

Chen, L. C., Papandreou, G., Kokkinos, I., Murphy, K., \& Yuille, A. L. (2017). Deeplab: Semantic image segmentation with deep convolutional nets, atrous convolution, and fully connected crfs. IEEE transactions on pattern analysis and machine intelligence, 40(4), pp. 834-848. arXiv:1606.00915

Choy, C., Gwak, J., \& Savarese, S. (2018). 4D spatio-temporal convnets: Minkowski convolutional neural networks. CVPR.

Dai, A., Chang, A. X., Savva, M., Halber, M., Funkhouser, T., \& Nießner, M. (2017). Scannet: Richly-annotated 3d reconstructions of indoor scenes. Proceedings of the IEEE Conference on Computer Vision and Pattern Recognition, pp. 5828-5839. arXiv:1702.04405

Dhanachandra, N., Manglem, K., \& Chanu, Y. J. (2015). Image segmentation using k-means clustering algorithm and subtractive clustering algorithm. Procedia Computer Science, 54, pp. 764-771. https://doi.org/10.1016/j.procs.2015.06.090

Dore, C., \& Murphy, M. (2017). Current state of the art of historical building information modelling. The International Archives of the Photogrammetry, Remote Sensing and Spatial Information Sciences, XLII-2/W5(5), $185-192$. https://doi.org/10.5194/isprsarchives-XLII-2-W5-185-2017.

Grilli, E., \& Remondino, F. (2019). Classification of 3D Digital Heritage. Remote Sensing, $11(7), 847$. https://doi.org/10.3390/rs11070847

Grilli, E., Menna, F., \& Remondino, F. (2017). A review of point cloud segmentation and classification algorithms. Int. Arch. Ph. Remote Sens. Spatial Inf. Sci., XLII-2/W3, 339-344. https://doi.org/10.5194/isprs-archives-XLII-2-W3-339-2017

Grussenmeyer, P., Landes, T., Voegtle, T., \& Ringle, K. (2008). Comparison Methods of Terrestrial Laser Scanning, Photogrammetry and Tacheometry Data for Recording of Cultural Heritage Buildings. International Archives of Photogrammetry, Remote Sensing and Spatial Information Sciences. 37.

Guo, Y., Wang, H., Hu, Q., Liu, H., Liu, L., \& Bennamoun, M. (2020). Deep Learning for 3D Point Clouds: A Survey. IEEE transactions on pattern analysis and machine intelligence. arXiv:1912.12033

Hackel, T., Savinov, N., Ladicky, L., Wegner, J., Schindler, K., \& Pollefeys, M. (2017). Semantic3d. net: a new large-scale point cloud classification benchmark. ISPRS Annals of Photogrammetry, Remote Sensing and Spatial Information Sciences, pp. 91-98. arXiv:1704.03847

He, K., Zhang, X., Ren, S., \& Sun, J. (2016). Deep residual learning for image recognition. Proceedings of the IEEE conference on computer vision and pattern recognition, 770-778. arXiv:1512.03385 
Heipke, C., \& Rottensteiner, F. (2020). Deep learning for geometric and semantic task in photogrammetry and remore sensing. Geo-spatial Information Science, 23(1), 10-19. https://doi.org/10.1080/10095020.2020.1718003

Hu, Q., Yang, B., Xie, L., Rosa, S., Guo, Y., Wang, Z., Trigoni, N., \& Markham, A. (2020). RandLA-Net: Efficient semantic segmentation of large-scale point clouds. CVPR. arXiv:1911.11236

Hua, B. S., Tran, M. K., \& Yeung, S. K. (2018). Pointwise convolutional neural networks. CVPR. arXiv:1712.05245

landola, F. N., Han, S., Moskewicz, M. W., Ashraf, K., Dally, W. J., \& Keutzer, K. (2016). SqueezeNet: Alexnet-level accuracy with 50x fewer parameters and < $0.5 \mathrm{MB}$ model size," ICLR. arXiv:1602.07360

Khalil, A., \& Stravoravdis, S. (2019). H-BIM and the domains of data investigations of heritage buildings current state of art. International Archives of the Photogrammetry, Remote Sensing \& Spatial Information Sciences, XLII-2/W11, 661-667. https://doi.org/10.5194/isprs-archives-XLII-2-W11-661-2019.

Krizhevsky, A., Sutskever, I., \& Hinton, G. E. (2012) ImageNet classification with deepconvolutional neural networks. Advances in Neural InformationProcessing Systems, 2012, pp. 1097-1105. https://doi.org/10.1145/3065386

Laing, R., Leon, M., Isaacs, J., \& Georgiev, D. (2015). Scan to BIM: the development of a clear workflow for the incorporation of point clouds within a BIM environment. WIT Transactions on The Built Environment, 149, 279-288. https://doi.org/10.2495/BIM150241. https://doi.org/10.2495/BIM150241

Llamas, J., M. Lerones, P., Medina, R., Zalama, E., \& Gómez-García-Bermejo, J. (2017). Classification of Architectural Heritage Images Using Deep Learning Techniques. Appl. Sci., 7, 992. https://doi.org/10.3390/APP7100992

Lawin, F. J., Danelljan, M., Tosteberg, P., Bhat, G., Khan, F. S., \& Felsberg, M. (2017). Deep projective 3D semantic segmentation. CAIP. arXiv:1705.03428

Li, Z., Gan, Y., Liang, X., Yu, Y., Cheng, H., \& Lin, L. (2016). LSTM-CF: Unifying Context Modeling and Fusion with LSTMs for RGB-D Scene Labeling. Springer International Publishing, Cham, pp. 541-557. arXiv:1604.05000

Liu, F., Li, S., Zhang, L., Zhou, C., Ye, R., Wang, Y., \& Lu, J. (2017). 3DCNN-DQN-RNN: A deep reinforcement learning framework for semantic parsing of large-scale 3D point clouds. ICCV. arXiv:1707.06783

Liu, W., Rabinovich, A., \& Berg, A. C. (2015). Parsenet: Looking Wider to See Better. arXiv:1506.04579.

Long, J., Shelhamer, E., \& Darrell, T. (2015). Fully convolutional networks for semantic segmentation. Proceedings of the IEEE conference on computer vision and pattern recognition, 3431-3440. arXiv:1411.4038

López, F.J., Lerones, P.M., Llamas, J., Gómez-García-Bermejo, J., \& Zalama, E. (2018). A Review of Heritage Building Information Modeling (H-BIM). Multimodal Technologies and Interaction, 2, 21. https://doi.org/ 10.3390/mti2020021.

Macher, H., Landes, T., \& Grussenmeyer, P. (2015). Point clouds segmentation as a base for as-built BIM creation. ISPRS Annals of the Photogrammetry, Remote Sensing and Spatial Information Sciences, Volume II-5/W3.

Matrone, F.; Grilli, E.; Martini, M.; Paolanti, M.; Pierdicca, R.; \& Remondino, F. (2020a). Comparing Machine and Deep Learning Methods for Large 3D Heritage Semantic Segmentation. ISPRS Int. J. Geo-Inf., 9, 535.

Matrone, F., Lingua, A., Pierdicca, R., Malinverni, E., Paolanti, M., Grilli, E., Remondino, F., Murtiyoso, A., \& Landes, T. (2020b). A benchmark for large-scale heritage point cloud semantic segmentation. ISPRS - International Archives of the Photogrammetry, Remote Sensing and Spatial Information Sciences. XLIII-B2. 1419-1426. https://doi.org/10.5194/isprs-archives-XLIII-B2-2020-1419-2020.

Maturana, D., \& Scherer, S. (2015). Voxnet: A 3d convolutional neural network for real-time object recognition. IEEE/RSJ International Conference on Intelligent Robots and Systems (IROS), pp. 922-928. https://doi.org/10.1109/IROS.2015.7353481

Meng, H. Y., Gao, L., Lai, Y., \& Manocha, D. (2018). Vv-net: Voxel vae net with group convolutions for point cloud segmentation. arXiv preprintarXiv:1811.04337.

Milioto, A., Vizzo, I., Behley, J., \& Stachniss, C. (2019). RangeNet++: Fast and accurate lidar semantic segmentation. IROS.

Minaee, S., Boykov, Y., Porikli, F., Plaza, A.J., Kehtarnavaz, N., \& Terzopoulos, D. (2020). Image Segmentation Using Deep Learning: A Survey. ArXiv, abs/2001.05566.

Minaee S., \& Wang, Y. (2019). An admm approach to masked signal decomposition using subspace representation. IEEE Transactions on Image Processing, 28, no. 7, pp. 3192-3204. arXiv:1704.07711v2

Murphy, M., Mcgovern, E., \& Pavia, S. (2009). Historic building information modelling (HBIM). Structural Survey, 27(4), 311-327. https://doi.org/10.1108/02630800910985108.

Nguyen, A., \& Le, B. (2013). 3D point cloud segmentation: A survey. 6th IEEE conference on robotics, automation and mechatronics(RAM), 225-230. https://doi.org/10.1109/RAM.2013.6758588

Nock, R., \& Nielsen, F. (2004). Statistical region merging. IEEE Transactions on pattern analysis and machine intelligence, 26(11), pp. 1452-1458. https://doi.org/10.1109/TPAMI.2004.110 
Oreni, D. (2013). From 3D content models to H-BIM for conservation and management of built heritage. Computational science and its applications. ICCSA 2013. lecture notes in computer science, 7974 . https://doi.org/10.1007/978-3642-39649

Paszke, A., Chaurasia, A., Kim, S., \& Culurciello, E. (2015). Enet: A Deep Neural Network Architecture for Real-Time Semantic Segmentation. arXiv:1606.02147.

Qi, C. R., Su, H. Mo, K., \& Guibas, L. J. (2017). PointNet: Deep learning on point sets for 3D classification and segmentation. CVPR. arXiv:1612.00593

Qi, C. R., Yi, L., Su, H., \& Guibas, L. J. (2017). PointNet++: Deep hierarchical feature learning on point sets in a metric space. NeurlPS. arXiv:1706.02413

Riegler, G., Osman Ulusoy, A., \& Geiger, A. (2017). Octnet: Learning deep 3d representations at high resolutions. Proceedings of the IEEE Conference on Computer Vision and Pattern Recognition, pp. 3577-3586.

Rocha, G., Mateus, L., Fernández, J., \& Ferreira, V. (2020). A Scan-to-BIM Methodology Applied to Heritage Buildings. Heritage, 3(1), 47-67. https://doi.org/10.3390/heritage3010004.

Rodriguez, C., Reinoso, J. F., Rivas-Lopez, E., Gomez-Blanco, A., Ariza-Lopez F., \& Ariza, I. (2016). From point cloud to BIM : an integrated workflow for documentation, research and modelling of architectural heritage. Survey Review, 50(360), 1-20. https://doi.org/10.1080/00396265.2016.1259719.

Ronneberger, O., Fischer, P., \& Brox, T. (2015). U-Net: convolutional networks forbiomedical image segmentation. Medical Image Computing andComputer-Assisted Intervention (MICCAl), 9351 of LNCS, pp. 234-241.

Rosu, R. A., Schutt, P., Quenzel, J., \& Behnke, S. (2019). LatticeNet: Fast point cloud segmentation using permutohedral lattices. arXiv preprint arXiv:1912.05905. arXiv:1409.1556

Simonyan, K., \& Zisserman, A. (2014). Very Deep Convolutional Networks forLarge-Scale Image Recognition. arXiv:1409.1556.

Su, H., Maji, S., Kalogerakis, E., \& Learned-Miller, E. (2015). Multi-viewconvolutional neural networks for 3d shape recognition. Proceedings on the IEEE international conference on computer vision, 945-953.

Su, H., Jampani, V., Sun, D., Maji, S., Kalogerakis, E., Yang, M. H., \& Kautz, J. (2018). SplatNet: Sparse lattice networks for point cloud processing. CVPR. arXiv:1802.08275

Szegedy, C., Liu, W., Jia, Y., Sermanet, P., Reed, S., Anguelov, D., Erhan, D., Vanhoucke, V., \& Rabinovich, A. (2015). Going deeper with convolutions, Proceedings of the IEEE Conference on Computer Vision and Pattern Recognition 1-9. arXiv:1409.4842

Tang, P., Huber, D., Akinci, B., Lipman, R., \& Lytle, A. (2010). Automatic reconstruction of as-built building information models from laserscanned point clouds: A review of related techniques. Automation in construction, 19(7), 829-843.

Tchapmi, L., Choy, C., Armeni, I., Gwak, J., \& Savarese, S. (2017). Segcloud: Semantic segmentation of 3d point clouds. 2017 International Conference on 3D Vision (3DV), pp. 537-547, IEEE. arXiv:1710.07563

Ulku, I., \& Akagunduz, E. (2019). A Survey on Deep Learning-based Architectures for Semantic Segmentation on 2D images. Computer Vision and Pattern Recognition. arXiv:1912.10230

Varney, N., Asari, V. K., \& Graehling, Q. (2020). Dales: A large-scale aerial lidar data set for semantic segmentation," arXiv preprintarXiv:2004.11985.

Volk, R., Stengel, J., \& Schultmann, F. (2014). Building Information Modelling (BIM) for existing buildings - Literature review and future needs. Automation in Construction. Elsevier B.V., 38, 109-127. https://doi.org/10.1016/j.autcon.2013.10.023.

Vosselman, G., Coenen, M., \& Rottensteiner, F. (2017). Contextual segmentbased classification of airborne laser scanner data. ISPRS journal of photogrammetry and remote sensing, $128,354-371$. https://doi.org/10.1016/j.isprsjprs.2017.03.010

Weinmann, M., Jutzi, B., Hinz, S., \& Mallet, C. (2015). Semantic point cloud interpretation based on optimal neighborhoods, relevant features and efficient classifiers. ISPRS Journal of Photogrammetry and Remote Sensing, 105, $286-304$.

Weinmann, M., Schmidt, A., Mallet, C., Hinz, S., Rottensteiner, F., \& Jutzi, B. (2015). Contextual classification of point cloud data by exploiting individual 3d neigbourhoods. ISPRS Annals of the Photogrammetry, Remote Sensing and Spatial Information Sciences II-3, 4(2), 271-278. https://doi.org/10.5194/isprsannals-II-3-W4-271-2015

Wang Y., Sun, Y., Liu, Z., Sarma, S. E., Bronstein, M. M., \& Solomon, J. M. (2018). Dynamic graph cnn for learning on point clouds. arXivpreprint arXiv:1801.07829.

Wang, S., Suo, S., Ma, W. C., Pokrovsky, A., \& Urtasun, R. (2018). Deep parametric continuous convolutional neural networks. CVPR. arXiv:2101.06742

Xie, Y., Tian, J. \& Zhu, X. (2020). Linking Points with Labels in 3D: A Review of Point Cloud Semantic Segmentation. IEEE Geoscience and Remote Sensing Magazine. https://doi.org/10.1109/MGRS.2019.2937630. 
Yu, H., Tan, Y., Wang, L., Yaonan, S., Sun, W., Mi, \& Yandong, M. T. (2018). Methods and Datasets on Semantic Segmentation: A review. Neurocomputing, 304. https://doi.org/10.1016/j.neucom.2018.03.037.

Zhang, J., Zhao, X., Chen, Z., \& Lu, Z. (2019). A Review of Deep Learning-Based Semantic Segmentation for Point Cloud. IEEE Access, 7, pp. 179118-179133. doi: 10.1109/ACCESS.2019.2958671.

Zhu, X., Tuia, D., Mou, T., Xia, G. S., Zhang, L., Xu, F., \& Fraundorfer, F. (2017). Deep learning in remote sensing: A comprehensive review and list of resources. IEEE Geoscience and Remote Sensing Magazine, 5(4), 8-12 